\title{
Finite difference simulation of diffusion behaviors under inter- and intra-molecular multiple-quantum coherences in liquid NMR
}

\author{
Congbo Cai ${ }^{\text {a }}$, Zhong Chen ${ }^{\mathrm{a}, \mathrm{c}, *}$, Shuhui Cai ${ }^{\mathrm{a}}$, Lian-Pin Hwang ${ }^{\mathrm{b}}$, Jianhui Zhong ${ }^{\mathrm{c}}$ \\ ${ }^{a}$ Department of Physics, State Key Laboratory of Physical Chemistry of Solid Surface, Xiamen University, Xiamen 361005, PR China \\ b Department of Chemistry, National Taiwan University, and Institute of Atomic and Molecular Science, Academia Sinica, Taipei, Taiwan \\ ${ }^{\mathrm{c}}$ Departments of Radiology and Physics and Astronomy, University of Rochester, Rochester, NY 14642, USA
}

Received 20 January 2005; in final form 28 March 2005

Available online 18 April 2005

\begin{abstract}
The behaviors of molecular self-diffusion were simulated in complex spin systems with both intra-molecular scalar couplings and inter-molecular dipolar couplings in liquid nuclear magnetic resonance (NMR). The simulation algorithm was based on a combination of the non-linear Bloch equations, product operator matrix, and finite difference method. The simulated results reveal different diffusion behaviors of inter- and intra-molecular multiple-quantum coherences, coincident with theoretical predictions and experimental measurements. Compared with the Monte Carlo method, the finite difference method is more precise and efficient for simulating diffusion behaviors of multiple-quantum coherences.
\end{abstract}

(c) 2005 Elsevier B.V. All rights reserved.

\section{Introduction}

Translational diffusion is one of the most fundamental forms of transport in chemical and biological systems, and responsible for all chemical reactions [1]. Diffusion nuclear magnetic resonance (NMR) spectroscopy and diffusion-weighted magnetic resonance imaging (MRI) are presently the only available non-invasive methods that provide information of molecular displacements in a spatial scale comparable to cell dimensions of biological systems [2]. In diffusion NMR spectroscopy and diffusion-weighted MRI, pulsed field gradients have long been recognized as a useful means of probing migration of nuclear spins, inter-molecular interactions, and selfaggregation [3-7]. When a two-pulse sequence separated by a time interval is applied to a highly polarized system, multiple spin echoes (MSEs) can be observed in the pres-

\footnotetext{
* Corresponding author. Fax: +86 5922189426.

E-mail address: chenz@jingxian.xmu.edu.cn (Z. Chen).
}

ence of magnetic field gradients, as seen in solid ${ }^{3} \mathrm{He}$, liquid ${ }^{3} \mathrm{He}$, and water [8]. These phenomena have been explained with the theories based on either inter-molecular multiple-quantum coherences (iMQCs) or dipolar field [9]. Recent studies on the iMQCs have resulted in several exciting novel applications, such as high-resolution NMR spectra in inhomogeneous field [10] and new contrast mechanism in in vivo MRI [11]. Recently, we reported that iMQCs have unconventional diffusion behaviors due to different physical mechanism from intra-molecular MQCs [12,13].

Although the diffusion behavior under the effect of dipolar field has been discussed previously [14,15], the mathematics required for describing diffusion is rather complicated except for the free diffusion or restricted diffusion in samples with certain simple geometries. As a result, analytical solutions are generally unavailable and numerical solutions are called for. Blanton provided a powerful NMR tool kit to simulate almost any NMR explorations either experimental or theoretical in nature 
[16], but diffusion effect is not included. Warren and coworkers $[17,18]$ provided an effective tool based on the Torrey's method in $k$-space to simulate the diffusion behaviors of spin systems such as $\mathrm{H}_{2} \mathrm{O}$ under dipolar fields. Recently, we employed the iMQC model and Monte Carlo method to simulate the diffusion behaviors of iMQCs during the evolution period of the CRAZED sequence for simple spin systems such as $\mathrm{H}_{2} \mathrm{O}$ [19]. This simulation algorithm takes substantial computational time and does not take into account the intra-molecular scalar couplings. As an improvement, we proposed a method combining the non-linear Bloch equations, product operator matrix, and Monte Carlo method to simulate the diffusion behaviors under dipolar fields with intra-molecular scalar couplings [20]. However, it is still very time-consuming for complex spin systems under multiple pulse sequences.

Besides the Monte Carlo method [21] and Torrey's method in $k$-space [17], other numerical methods such as finite difference [22] and finite element [23] have also been used to study the diffusion behaviors in liquid NMR. Although the Torrey's method in $k$-space is less time consuming and more effective than the finite element method [24], it still takes much longer computational time than the finite difference method when the restricted diffusion next to boundaries is considered [25]. The Monte Carlo method also costs much longer computational time than the finite difference method although it is more flexible and intuitive. Moreover, it often results in lower precision. Therefore, the finite difference method possesses the advantage of taking least computational time among the numerical methods mentioned above. It also allows great flexibility in assessing complex structures such as what existed in lungs in diffusion-weighted MRI [26].

In this Letter, a finite difference method was applied to a combined model with the product operator matrix and non-linear Bloch equations to simulate the intraand inter-molecular MQC diffusions. For comparison, the Monte Carlo method was also utilized in place of the finite difference method for the same systems.

\section{Simulation method}

The motion equation of magnetization vector $\boldsymbol{M}^{(i)}(\boldsymbol{r}, t)$ in a frame rotating at the Larmor frequency is given by:

$$
\begin{aligned}
\frac{\mathrm{d} \boldsymbol{M}^{(i)}(\boldsymbol{r}, t)}{\mathrm{d} t}= & \gamma \boldsymbol{M}^{(i)}(\boldsymbol{r}, t) \times\left\{\frac{\omega_{i}}{\gamma} \hat{\boldsymbol{z}}+\boldsymbol{G}(\hat{\boldsymbol{s}}, \boldsymbol{r}) z+\Delta \boldsymbol{B}(\boldsymbol{r}, t)\right. \\
& \left.+\sum_{j}\left[\boldsymbol{B}_{r}^{(j)}(\boldsymbol{r}, t)+\boldsymbol{B}_{d}^{(j)}(\boldsymbol{r}, t)\right]\right\} \\
& -\frac{M_{x}^{(i)}(\boldsymbol{r}, t) \hat{\boldsymbol{x}}-M_{y}^{(i)}(\boldsymbol{r}, t) \hat{\boldsymbol{y}}}{T_{2}^{(i)}(\boldsymbol{r})}+\frac{M_{0}^{(i)} \hat{\boldsymbol{z}}-M_{z}^{(i)}(\boldsymbol{r}, t) \hat{\boldsymbol{z}}}{T_{1}^{(i)}(\boldsymbol{r})} \\
& +D_{T}^{(i)} \nabla^{2} \boldsymbol{M}^{(i)}(\boldsymbol{r}, t),
\end{aligned}
$$

where $\gamma$ is the gyromagnetic ratio; $\hat{\boldsymbol{x}}, \hat{\boldsymbol{y}}$, and $\hat{\boldsymbol{z}}$ are unit vectors along the Cartesian coordinate axes; the superscript $i$ represents the $i$ th spin species; $G(\hat{\boldsymbol{s}}, \boldsymbol{r})$ is the strength of gradients applied for modulating the dipolar field along direction $\hat{\boldsymbol{s}}$ at position $\boldsymbol{r}$, where $\hat{\boldsymbol{s}}$ is a unit vector; $\Delta \boldsymbol{B}(\boldsymbol{r}, t)$ is the inhomogeneous field at position $\boldsymbol{r}$; $D_{\mathrm{T}}$ is the translational self-diffusion coefficient; $T_{1}$ and $T_{2}$ are the longitudinal and transverse relaxation times, respectively; $\boldsymbol{M}^{(i)}(\boldsymbol{r}, 0)=M_{0}^{(i)} \hat{\boldsymbol{z}}$ is the initial magnetization vector; $\boldsymbol{B}_{r}(\boldsymbol{r}, t)$ is the radiation damping field, which is fed back to the spins through the induced free-induction-decay (FID) current in the receiver coil [27]. $\boldsymbol{B}_{\mathrm{d}}(\boldsymbol{r}, t)$ is the dipolar field at time $t$ and position $\boldsymbol{r}$. The effect of diffusion during the evolution period can generally be ignored when the evolution time is short. However, when the observable signal evolves over a period equivalent to a few multiples of the dipolar demagnetizing time $\tau_{\mathrm{d}}=1 /\left(\gamma \mu_{0} M_{0}\right)$, it becomes essential to include the diffusion effect during the acquisition time [28].

The effects of relaxation, diffusion, chemical shift, radiation damping, and long-range dipolar field can all be incorporated into spin dynamic calculations by the non-linear Bloch equations. However, the Bloch equations fail to describe magnetization vectors when scalar couplings exist in spin systems. Though the density matrix formalism can deal with complicated quantum mechanical behaviors such as scalar couplings, it is difficult to handle macroscopic effects such as radiation damping, diffusion, and long-range dipolar field. In our recent report [20], a product operator matrix, $M_{\text {mat }}$, was proposed to describe the evolution behaviors of scalar couplings in the frame of the non-linear Bloch equations. The evolution of the elements of product operator matrix under chemical shift, radio-frequency (RF) field, inhomogeneous background field, dipolar field, radiation damping, and diffusion can be described by the non-linear Bloch equations shown in Eq. (1). After every evolution of the Cash-Karp Runge-Kutta equation, the corresponding scalar coupling process is calculated [20].

The diffusion part of the non-linear Bloch equations can be solved by the finite difference method, which can be implemented in two simple steps for one-dimension diffusion [26]:

$$
\begin{aligned}
& M_{j}^{\prime}=M_{j}^{n}+D_{j-1, j} \frac{\Delta t}{\Delta x^{2}}\left(M_{j-1}^{n}-M_{j}^{n}\right), \\
& M_{j}^{n+1}=M_{j}^{\prime}+D_{j, j+1} \frac{\Delta t}{\Delta x^{2}}\left(M_{j+1}^{n}-M_{j}^{n}\right),
\end{aligned}
$$

where $M_{j}^{n}$ is the magnetization at the $j$ th position along a special axis at $n$th time step; $\Delta x$ and $\Delta t$ are spatial and time step sizes, respectively; $M_{j}^{n} \equiv M(t, x)$ with $t=n \Delta t$ and $x=j \Delta x . M_{j}^{\prime}$ is an intermediate variable between the $n$th and $(n+1)$ th time intervals; $D_{j, j+1}$ represents 
the effective diffusion coefficient between the $j$ th and $(j+1)$ th elements. A detailed discussion can be found in [26].

To calculate the diffusion behaviors by the finite difference method, every element of the product operator matrix is operated directly in Eqs. (2) and (3) by substituting $M_{j}^{n}$ with $M_{\text {mat }}\left(I_{1 a}, I_{2 a}, \ldots, I_{i a}, \cdots, I_{n+m, a}\right)$. Thus, the simulation algorithm is simple and efficient for combining the finite difference method and product operator matrix.

\section{Experimental and simulated processes}

All ${ }^{1} \mathrm{H}$ NMR experiments were recorded on a Varian Unity+ $500 \mathrm{MHz}$ NMR spectrometer at room temperature. For inter-molecular double-quantum and zeroquantum coherence (iDQC and $\mathrm{iZQC}$ ) experiments, the sample was a concentrated solution of $\mathrm{CHBr}_{2} \mathrm{CH}_{2} \mathrm{Br}$ in acetone, where $\mathrm{CHBr}_{2} \mathrm{CH}_{2} \mathrm{Br}$ corresponded to an $A X_{2}$ system with $\omega_{A}=278.7 \mathrm{~Hz}, \omega_{X}=-688.7 \mathrm{~Hz}$, $J_{A X}=5.7 \mathrm{~Hz}$, and the protons in acetone was denoted as $C$ with $\omega_{C}=0 \mathrm{~Hz}$ for convenience. For intra-molecular double quantum coherence (DQC) experiments, the solvent was $\mathrm{CDCl}_{3}$, and the solution $\left(\mathrm{CHBr}_{2} \mathrm{CH}_{2} \mathrm{Br}\right)$ was dilute. The chemical shifts of the solute changed to $\omega_{A}=324.0 \mathrm{~Hz}, \omega_{X}=-736.1 \mathrm{~Hz}$ relative to the same reference as in the iMQC experiments (i.e., the chemical shift of acetone was zero) due to different solvent effects. The scalar coupling constant is still $J_{A X}=5.7 \mathrm{~Hz}$.

Macroscopic background gradients due to susceptibility differences at the sample interfaces and radiation damping can lead to the seemingly strange effect of the echo signal growing as the gradient strength increases at low applied gradient strengths [4]. To reduce their effects, the following precautions in diffusion measurements were taken: (1) the effect of radiation damping was effectively suppressed by detuning the probe; (2)

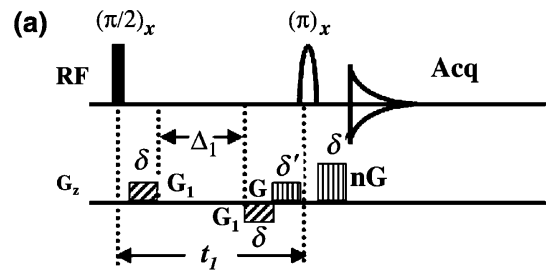

(b)

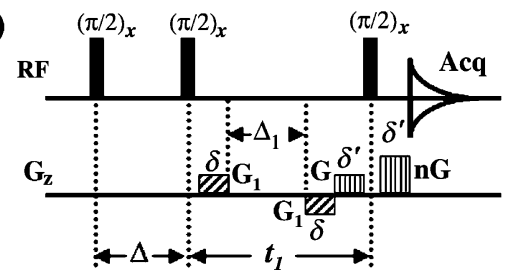

Fig. 1. Pulse sequences used in this Letter: (a) a modified CRAZED sequence for iDQCs $(\alpha=\pi / 2$ and $n=-2)$, iZQCs $(\alpha=\pi / 2$ and $n=0)$ and conventional SQCs $(\alpha=0$ and $G=0)$. The second RF pulse was set to be selective only for solvent to eliminate the solute-solute iMQCs; (b) a modified sequence for intra-molecular DQCs $(n= \pm 2)$ with $\Delta=0.08 \mathrm{~s}$. For both (a) and (b), the amplitude of coherenceselection gradients $G$ was $10 \mathrm{G} / \mathrm{cm}, \delta=2 \mathrm{~ms}$, and $\Delta_{1}=0.1 \mathrm{~s}$.

the magnetic field was shimmed carefully so that the line-width of a conventional 1D SQC spectrum is smaller than $1.0 \mathrm{~Hz}$; (3) the diffusion weighted gradients were begun from a relative high strength, so the effect of background gradients is quite small compared to that of the diffusion weighted gradients. Since we used standard $5 \mathrm{~mm}$ NMR tubes in the experiments, the effect of susceptibility match was not taken into account. Therefore, susceptibility differences at the sample interfaces may lead to some experimental errors [29].

The field gradients applied during the spin evolution attenuate NMR signals. When the pulse sequences shown in Fig. 1 are employed, the diffusion attenuation factor $E$ due to free diffusion is given by:

$$
E=E_{0} \mathrm{e}^{-\gamma^{2} G_{1}^{2} \delta^{2} D_{\mathrm{T}}^{\mathrm{App}}\left(\Delta_{1}+2 \delta / 3\right)},
$$

Table 1

Diffusion coefficients of iMQCs obtained from theoretical predictions, experiments, and simulations for the concentrated solution

\begin{tabular}{|c|c|c|c|c|c|}
\hline \multirow[t]{2}{*}{ Diffusion rates $\left(10^{-9} \mathrm{~m}^{2} / \mathrm{s}\right)$} & \multicolumn{2}{|l|}{ SQC } & \multicolumn{2}{|c|}{$\mathrm{iDQC}(n=-2)$} & \multirow{2}{*}{$\frac{\mathrm{iZQC}}{D_{\mathrm{iZQC}}^{A X, C}}$} \\
\hline & $D_{\mathrm{SQC}}^{C}$ & $D_{\mathrm{SQC}}^{A X}$ & $\overline{D_{\mathrm{iDQC}}^{C, C}}$ & $D_{\mathrm{iDQC}}^{A X, C}$ & \\
\hline Theory & 2.32 & 1.68 & 4.64 & 4.00 & 4.00 \\
\hline Experiment & $2.32 \pm 0.10$ & $1.68 \pm 0.08$ & $4.76 \pm 0.25$ & $3.89 \pm 0.19$ & $3.85 \pm 0.18$ \\
\hline Finite difference & $2.31 \pm 0.01$ & $1.68 \pm 0.01$ & $4.63 \pm 0.01$ & $3.99 \pm 0.01$ & $3.99 \pm 0.01$ \\
\hline Monte Carlo & $2.40 \pm 0.03$ & $1.77 \pm 0.02$ & $4.72 \pm 0.06$ & $3.96 \pm 0.05$ & $3.99 \pm 0.06$ \\
\hline
\end{tabular}

Table 2

Diffusion coefficients of intra-molecular MQCs obtained from theoretical predictions, experiments, and simulations for the dilute solution

\begin{tabular}{llll}
\hline Diffusion rates $\left(10^{-9} \mathrm{~m}^{2} / \mathrm{s}\right)$ & $D_{\mathrm{SQC}}^{A X}$ & $D_{\mathrm{DQC}}^{A X}(n=+2)$ & $D_{\mathrm{DQC}}^{A X}(n=-2)$ \\
\hline Theory & 7.85 & 31.40 & 31.40 \\
Experiment & $7.85 \pm 0.19$ & $29.82 \pm 0.51$ & $31.69 \pm 0.53$ \\
Finite difference & $7.91 \pm 0.01$ & $31.56 \pm 0.02$ & $31.52 \pm 0.02$ \\
Monte Carlo & $7.98 \pm 0.05$ & $31.87 \pm 0.10$ & $31.93 \pm 0.12$ \\
\hline
\end{tabular}


where $D_{\mathrm{T}}^{\mathrm{App}}$ is the apparent diffusion coefficient; $\delta$ and $G_{1}$ are the duration and amplitude of diffusion weighting gradients, respectively; $\Delta_{1}$ is the time interval between the two diffusion weighting gradients. It was assumed that the longitudinal and transversal relaxation times were all $1 \mathrm{~s}$, which are close to the experimental measurements. The length of the sample was $1 \mathrm{~cm}$ along the $z$ axis and was divided into $2000 \mathrm{U}$. The amplitude of coherence-selection gradient $G$ was $10 \mathrm{G} / \mathrm{cm}$. The total acquisition time of each FID signal was $4.0 \mathrm{~s}$ with a total acquisition number of 10000 . For the Monte Carlo method, the number of particles used in the simulation was 400000 to achieve results with high accuracy. All simulations were performed on a normal PC (AMD $\mathrm{XP} 2500+, 512 \mathrm{Mb}$ memories) and the program was written in Visual $\mathrm{C}++$.

The pulse sequence shown in Fig. 1a was used to measure the apparent diffusion rates of iDQCs, iZQCs, and conventional SQCs. The pulse sequence shown in Fig. 1b was used to measure and simulate the apparent diffusion rates of DQCs. The SQC diffusion coefficients of $C$ (acetone) and $A X_{2}\left(\mathrm{CHBr}_{2} \mathrm{CH}_{2} \mathrm{Br}\right)$ components in concentrated solution were measured experimentally and listed in Table 1. The diffusion coefficient of a dilute spin system of $A X_{2}\left(\mathrm{CHBr}_{2} \mathrm{CH}_{2} \mathrm{Br}\right)$ in $\mathrm{CDCl}_{3}$ solution was measured experimentally and listed in Table 2 . It shows that the diffusion coefficient of component $A X_{2}$ is greatly affected by solvent. The measured SQC diffusion coefficients were used as the theoretical values and physical diffusion rates of the simulated spins.

\section{Discussion}

The diffusion coefficients of iMQCs during the evolution period obtained from the theoretical predictions, experimental measurements, and finite difference simulation for the concentrated solution are listed in Table 1. The corresponding normalized diffusion attenuation fitting curves are shown in Fig. 2. The simulated SQC diffusion coefficients are very close to the experimental values. For iDQCs $(n=-2)$, both experimental and simulated results have the following relationships: $D_{\mathrm{iDQC}}^{A X, C}=D_{\mathrm{SQC}}^{C}+D_{\mathrm{SQC}}^{A X}$ and $D_{\mathrm{iDQC}}^{C, C}=2 D_{\mathrm{SQC}}^{C}$, where $D_{\mathrm{iDQC}}^{A X, C}$ denotes the apparent diffusion coefficient of the signal originating from the solute-solvent iDQCs, and $D_{\mathrm{iDQC}}^{C, C}$ represents the apparent diffusion coefficient of the signal originating from the solvent-solvent iDQCs. Similarly, both the experimental and simulated results satisfies the following relationship for iZQCs $(n=0)$ : $D_{\mathrm{iZQC}}^{A X, C}=D_{\mathrm{SQC}}^{C}+D_{\mathrm{SQC}}^{A X}$, where $D_{\mathrm{iZQC}}^{A X, C}$ denotes the apparent diffusion coefficient of the signal originating from the solute-solvent iZQCs. There is not signal originating from the solvent-solvent iZQCs because the second RF pulse is $\pi / 2$. In Table 1 , the simulated diffusion coefficients $D_{\mathrm{iDQC}}^{C, C}, D_{\mathrm{iDQC}}^{A X, C}$, and $D_{\mathrm{iZQC}}^{A X, C}$ are in good agreement
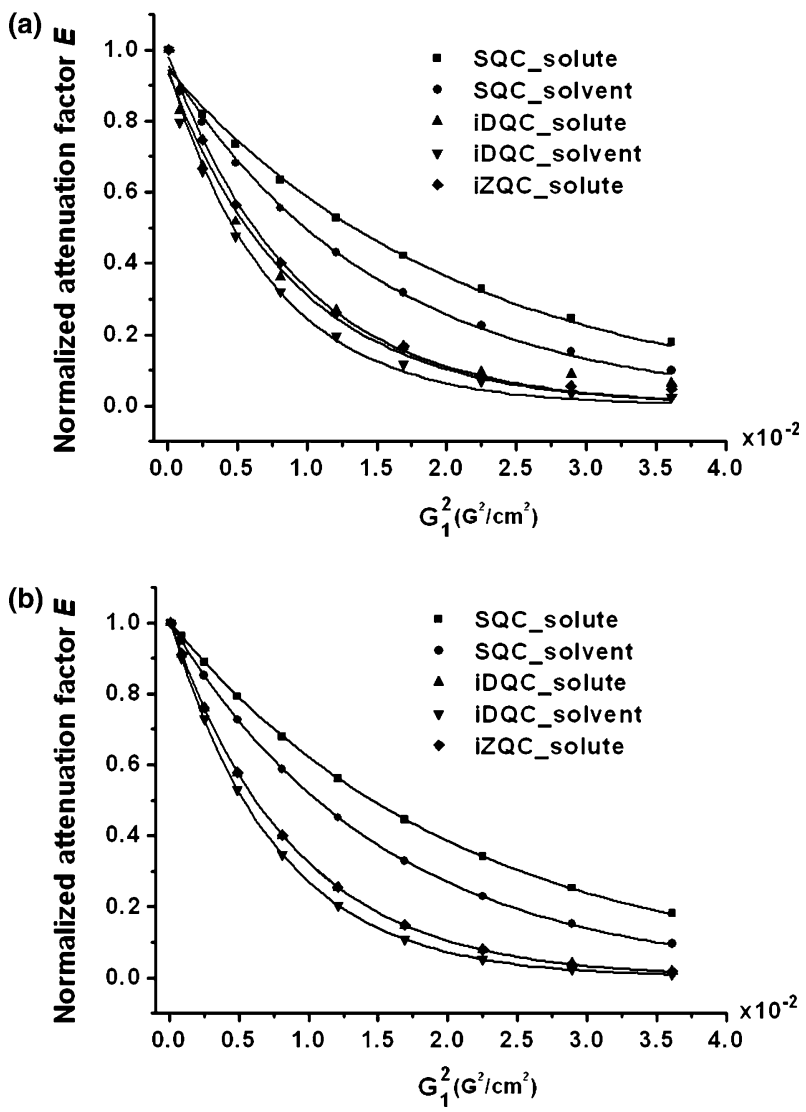

Fig. 2. Normalized diffusion attenuation fitting curves of conventional SQC and iMQC signals for concentrated solution: (a) experiment; (b) finite difference simulation. The curves denoted by ' $\mathbf{\Lambda}$ ' and ' ' were almost overlapped in (b). The duration of diffusion weighting gradient was $2 \mathrm{~ms}$, and the amplitude of diffusion weighting gradient $G_{1}$ was varied from 1 to $19 \mathrm{G} / \mathrm{cm}$ with an increment of $2 \mathrm{G} / \mathrm{cm}$.

with their theoretical predictions and experimental observations. The small deviations of experimental values may be due to the measured errors and non-ideal experimental conditions such as non-ideal RF pulses and the remnant radiation damping and background gradients.

According to the diffusion properties in a homo-nuclear experiment, the signals originated from the intramolecular MQCs would decay exponentially at a rate $D_{\mathrm{MQC}}=n^{2} D_{\mathrm{T}}[30]$, where $n$ is the order of MQCs. The apparent diffusion rates of the DQCs for dilute solution are given in Table 2. The corresponding normalized diffusion attenuation fitting curves based on the results of experiments and finite difference simulations are shown in Fig. 3. For $n= \pm 2$, the simulated apparent diffusion rates from the finite difference method of the solute $D_{\mathrm{DQC}}^{A X}$ are coincident with the theoretical and experimental values. Compared to the theoretical predictions, the deviations of simulated results are less than $1 \%$ and the maximal deviation of experimental measurements is about $5 \%$. 

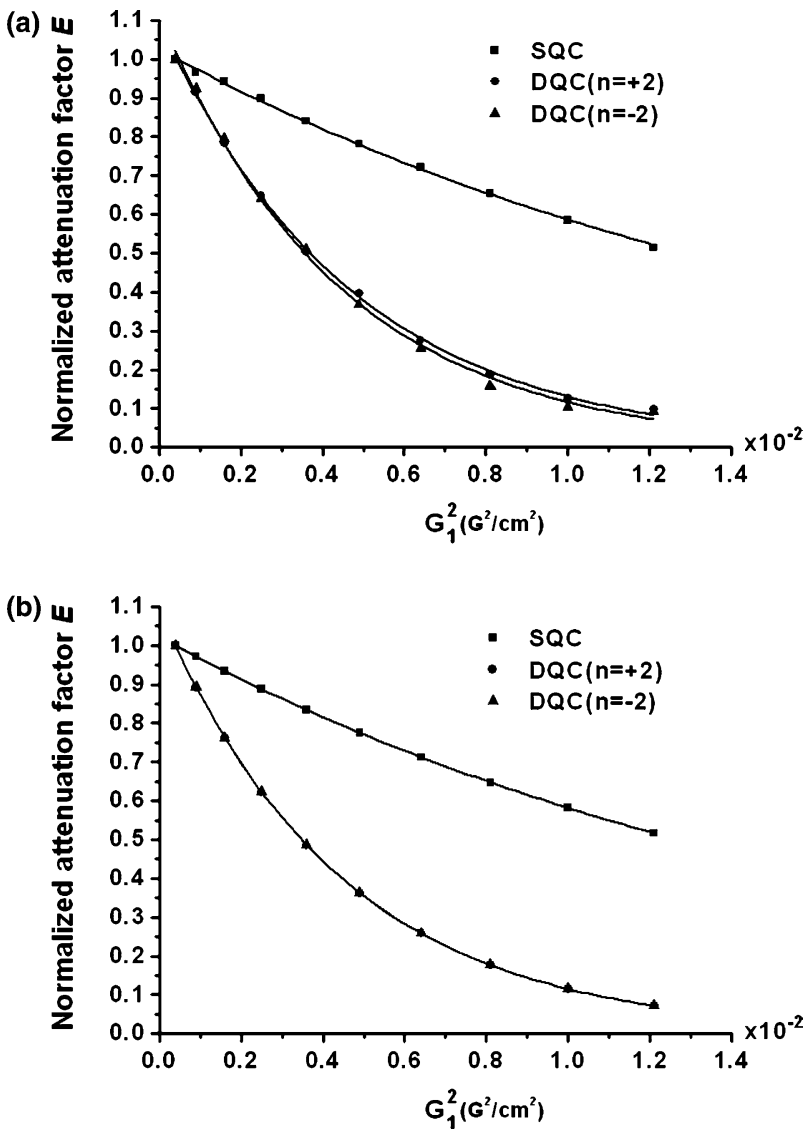

Fig. 3. Normalized diffusion attenuation fitting curves of conventional SQC and DQC signals for dilute solution: (a) experiment; (b) finite difference simulation. The curves denoted by ' $\boldsymbol{}$ ' and ' $\boldsymbol{\Delta}$ ' were almost overlapped in (b). The duration of diffusion weighting gradient was 1 $\mathrm{ms}$, and the amplitude of diffusion weighting gradient $\mathrm{G}_{1}$ was varied from 2 to $11 \mathrm{G} / \mathrm{cm}$ with an increment of $1 \mathrm{G} / \mathrm{cm}$.

To compare the simulation effects of the finite difference and Monte Carlo methods under the same condition for liquid NMR, the simulated results of SQCs, iDQCs and DQCs using the Monte Carlo method are also listed in Tables 1 and 2. The comparison of the simulated results shows that the finite difference method provides more precise results. Although the simulated results obtained from the Monte Carlo method are also reliable, its maximum errors from the theoretical predictions reach 5\%. On the other hand, the maximum errors of the simulated results are only $1 \%$ for the finite difference method. Furthermore, for SQC simulations, the finite difference method took only $150 \mathrm{~s}$ for a $1 \mathrm{D}$ spectrum, while the Monte Carlo method took about $11760 \mathrm{~s}$. The computational time of the former is only about $1.3 \%$ of the latter. For iDQC and DQC simulations, the finite difference method took only about $5.0 \%$ of the computing time of the Monte Carlo method. Obviously, the finite difference method saves much computational time compared to the Monte Carlo method. It is worth noting that the computational speed of the finite difference method de- pends on the pulse sequence greatly, while the computational speed of the Monte Carlo method is almost independent of the pulse sequence when the sampling time is the same. Because most of the elements in the product operator matrix are zero in SQC simulation, the simulation speed of SQCs using the finite difference method is much faster than that of other quantum coherences. While for the Monte Carlo method, much of the CPU time is spent on the random walk of particles. The calculation of the product operator matrix only takes a small part of the total computational time, so the computational speed is rather stable.

\section{Conclusion}

In this Letter, the finite difference and Monte Carlo methods were applied to simulate the diffusion behaviors of intra- and inter-molecular MQCs in combination with the product operator matrix and non-linear Bloch equations. The corresponding experiments and theoretical analyses were carried out to validate the simulation results. The results confirm the unconventional diffusion behaviors of iMQCs reported previously. More importantly, the results show that the combination of the finite difference method, non-linear Bloch equations, and product operator matrix provides a versatile tool for the study of the diffusion behaviors of complex spin systems with both inter-molecular dipolar couplings and intra-molecular scalar couplings under different pulse sequences. Compared with the Monte Carlo method, the finite difference method is more efficient and provides more precise results in liquid NMR simulation. They also show that the intra-molecular scalar couplings do not change the apparent diffusion rates of iMQCs. The simulation method proposed herein may be used to simulate the diffusion behaviors in more complicated cases, such as the diffusion in biologic tissue (e.g., lungs) with inter-molecular dipolar couplings and intra-molecular scalar couplings. The diffusion behaviors under the effect of radiation damping can also be studied with this method.

\section{Acknowledgment}

This work was supported by the NNSF of China under Grants 10234070, 10375049 and 10104011, and NIH under Grants NS41048.

\section{References}

[1] W.S. Price, Concepts Magn. Reson. 9 (1997) 299.

[2] S.D. Kennedy, J. Zhong, Magn. Reson. Med. 52 (2004) 1. 
[3] G.X. Lin, J.H. Zhang, H.H. Cao, A.A. Jones, J. Phys. Chem. B 107 (2003) 6179.

[4] W.S. Price, P. Stilbs, B. Jönsson, O. Söderman, J. Magn. Reson. 150 (2001) 49.

[5] S.Y. Huang, J.D. Walls, Y. Wang, W.S. Warren, Y.Y. Lin, J. Chem. Phys. 121 (2004) 6105.

[6] W.S. Price, H. Ide, Y. Arata, J. Phys. Chem. A 107 (2003) 4787.

[7] M.V. Giotto, J.H. Zhang, P.T. Inglefield, W.Y. Wen, A.A. Jones, Macromolecules 36 (2003) 4397.

[8] G. Deville, M. Bernier, J.M. Delrieux, Phys. Rev. B 19 (1979) 5666.

[9] S. Lee, W. Richter, S. Vathyam, W.S. Warren, J. Chem. Phys. 105 (1996) 874.

[10] Z. Chen, Z.W. Chen, J. Zhong, J. Am. Chem. Soc. 126 (2004) 446.

[11] T. Hou, Z. Chen, D.W. Hwang, J.H. Zhong, L.P. Hwang, Magn. Reson. Imag. 22 (2004) 543.

[12] Z. Chen, J. Zhong, J. Chem. Phys. 114 (2001) 5642.

[13] Z. Chen, Z.W. Chen, J. Zhong, J. Chem. Phys. 117 (2002) 8426.

[14] Z. Chen, Z.W. Chen, J. Zhong, J. Chem. Phys. 115 (2001) 10769.

[15] L. Ardelean, E. Kossel, R. Kimmich, J. Chem. Phys. 114 (2001) 8520.

[16] W.B. Blanton, J. Magn. Reson. 162 (2003) 269.
[17] S. Garrett-Roe, W.S. Warren, J. Magn. Reson. 146 (2000) 1.

[18] T. Enss, S. Ahn, W.S. Warren, Chem. Phys. Lett. 305 (1999) 101.

[19] Q.L. Lin, S.H. Cai, Z. Chen, J. Zhong, Chin. J. Chem. Phys. 17 (2004) 155.

[20] C.B. Cai, Z. Chen, S.H. Cai, J. Zhong, J. Magn. Reson. 172 (2005) 242.

[21] P.T. Callaghan, J. Magn. Reson. 129 (1997) 74.

[22] C.L. Chin, F.W. Wehrli, S.N. Hwang, D.L. Jaggard, D.B. Hackney, S.W. Wehrli, J. Magn. Reson. 160 (2003) 20.

[23] C. Mayer, A. Terheiden, J. Chem. Phys. 118 (2003) 2775.

[24] J.P. Marques, R. Bowtell, Proc. Intl. Soc. Magn. Reson. Med. 11 (2003) 1020.

[25] J.P. Marques, R. Bowtell, Proc. Intl. Soc. Magn. Reson. Med. 11 (2003) 1108.

[26] S. Fichele, M.N.J. Paley, N. Woodhouse, P.D. Griffiths, E.J.R.V. Beek, J.M. Wild, J. Magn. Reson. 167 (2004) 1.

[27] Y.Y. Lin, N. Lisitza, S. Ahn, W.S. Warren, Science 290 (2000) 118.

[28] C. Ramanathan, R. Bowtell, J. Chem. Phys. 114 (2001) 10854.

[29] K. Hayamizu, W.S. Price, J. Magn. Reson. 167 (2004) 328.

[30] D. Zax, A. Pines, J. Chem. Phys. 78 (1983) 6333. 